\title{
Kinematics of the 3(RPSP)-S Fully Spherical Parallel Manipulator by Means of Screw Theory
}

\author{
Jaime Gallardo-Alvarado * (D), Ramon Rodriguez-Castro, Luciano Perez-Gonzalez \\ and Carlos R. Aguilar-Najera \\ Department of Mechanical Engineering, Instituto Tecnologico de Celaya, TecNM, \\ Av. Tecnologico y A Garcia Cubas, Celaya 38010, Mexico; ramon.rodriguez@itcelaya.edu.mx (R.R.-C.); \\ luciano.perez@itcelaya.edu.mx (L.P.-G.); rafael.aguilar@itcelaya.edu.mx (C.R.A.-N.) \\ * Correspondence: jaime.gallardo@itcelaya.edu.mx; Tel.: +52-461-61-1-75-75; Fax: +52-461-61-1-79-79
}

Received: 16 May 2018; Accepted: 11 June 2018; Published: 15 June 2018

\begin{abstract}
In this work, the kinematics of a spherical parallel manipulator composed of three peripheral limbs equipped with linear actuators and a passive center shaft is approached by means of the theory of screws. The displacement analysis is carried out solving closure equations, which are obtained upon simple linear combinations of the components of two unit vectors describing the orientation of the moving platform. After, the input-output equations of velocity and acceleration of the spherical parallel manipulator are systematically obtained by resorting to reciprocal-screw theory. This strategy avoids the computation of the passive joint velocity and acceleration rates of the robot manipulator. Numerical examples illustrate the efficiency of the proposed method.
\end{abstract}

Keywords: parallel manipulator; spherical motion; Klein form; screw theory; kinematics

\section{Introduction}

One of the most important problems in spatial kinematics is the representation of spherical displacements and motions [1-7]. In robotics, usually this class of motion may be achieved using serial or parallel manipulators. However, the spherical motion can be performed also with a four bar spatially-closed kinematic chain with concentric revolute joints (4R) [8-12], which is the simplest version of a parallel manipulator.

A spherical parallel manipulator (SPM) is a mechanical device composed of a moving platform and a fixed platform connected to each other by means of two or more kinematic chains, the main virtue of which is that all points of the moving platform move on concentric spheres according to a point of the moving platform named the reference pole. Due to their large orientation workspace and compactness, SPMs have been widely employed as wrist joints $[13,14]$, camera-orienting devices $[15,16]$ and surgical robots [17-19]. On the other hand, the kinematics of spherical parallel manipulators has been extensively well studied and, without a doubt, approaching topics like inverse-forward displacement analysis, dexterity, workspace, detection of singularities, and so on [20-29]. In that concern, recently, Wu et al. [30] investigated the performance of an SPM generator of unlimited decoupled motions owing to its asymmetric architecture. Saafi et al. [31] approached the optimal joint torque distribution for a redundant SPM used as a haptic device for controlling a surgical robot. Arrouk et al. [32] improved the parametrization and representation of the orientation space of SPMs, as well as their assembly modes based on graphical approaches. Landuré and Gosselin [33] introduced a redundantly-actuated SPM equipped with linear actuators and an asymmetric architecture able to avoid singularities. Li et al. [34] designed a spherical motion generator integrating an electromagnetic actuator and the classical coaxial 3-dof spherical parallel manipulator. 
Enferadi and Shahi [35] introduced a 3(RPSP)-S spherical parallel manipulator, where R, P and S stand respectively for revolute, prismatic and spherical joint. In that paper, by a combination of two coupled trigonometric expressions and Rodrigues' rotation formula, the forward position analysis leads to a univariate polynomial equation of degree eight. In this work, the displacement analysis of this SPM is simplified by formulating closure equations based on two unit vectors representing the orientation of the moving platform, which leads to a system of six quadratic equations that are solved using the homotopy continuation method. This strategy avoids the computation of the rotation matrix. Furthermore, the instantaneous kinematics of the parallel manipulator is also investigated. To this end, the rest of the contribution is organized as follows. In Section 2, the topology of the SPM is briefly explained. The displacement analysis of the robot is carried out in Section 3. After, the input-output equation of the velocity of the SPM is systematically obtained by resorting to reciprocal-screw theory in Section 4. Following the trend of the velocity analysis, the input-output equation of acceleration is obtained in Section 5. With the purpose to illustrate the method, in Section 6, it is applied to numerical examples covering most of the issues treated in the contribution. Finally, some conclusions are given at the end of the paper.

\section{Architecture of the Spherical Parallel Manipulator}

In this section, the topology of the parallel manipulator (see Figure 1) is explained, as well as most of the notation used in the contribution.

The SPM is composed of three identical active peripheral kinematic chains and a passive center shaft connecting the moving platform to the fixed base through a spherical joint (S). Each external leg is connected to the fixed platform through a revolute joint $(\mathrm{R})$ followed by an actuated prismatic joint $(\underline{P})$ and ending with a compound spherical + prismatic joint $(\mathrm{S}+\mathrm{P})$ connecting the limb to the moving platform. In order to explain the geometry of the SPM, let us consider that $O_{-} X Y Z$ is a reference frame attached to the center $O$ of the fixed platform where the $Z$-axis is normal to the plane of the fixed platform. With this consideration, the nominal positions of the lower revolute joints are characterized by points $A_{i}$, located by vectors $\boldsymbol{a}_{i}$. Unless otherwise stated, in the rest of the contribution, $i=1,2,3$. The symmetry of the external limbs is such that the points $A_{i}$ form an equilateral triangle inscribed in a circle of radius $a$. Furthermore, the orientation of the revolute joints is defined by unit vectors $\hat{\boldsymbol{u}}_{i}$ lying in the plane of the moving platform pointing from $O$ to $A_{i}$. Meanwhile, the nominal positions of the compound joints are denoted by points $B_{i}$, located by vectors $\boldsymbol{b}_{i}$, where the $i$-th $(\mathrm{S}+\mathrm{P})$ compound joint slides along the axis of a unit vector $\hat{\boldsymbol{v}}_{i}$ pointing from $C$ to $B_{i}$ because of the prismatic joint. In that regard, $b_{i}$ represents the variable distance between $C$ and $B_{i}$. In order to achieve the spherical motion, the SPM requires three generalized coordinates, which are associated with the peripheral prismatic joints, a logical selection. The variable distance between points $A_{i}$ and $B_{i}$ is denoted as $q_{i}$.

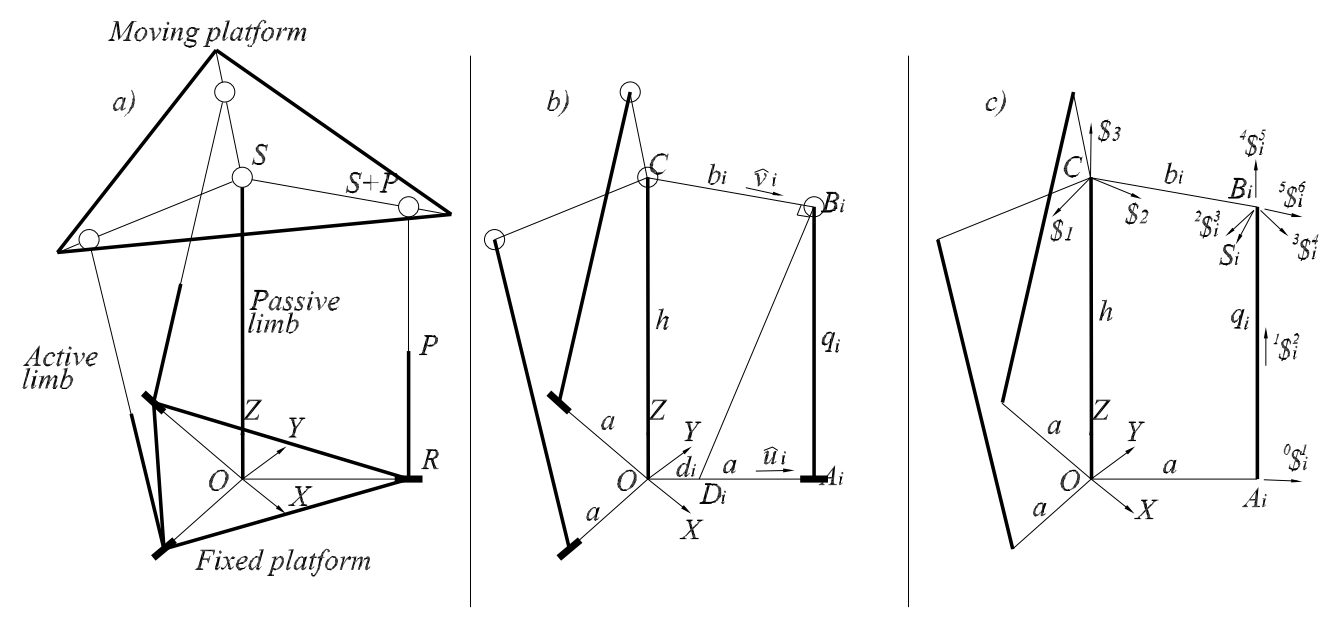

Figure 1. The spherical parallel manipulator. 


\section{Displacement Analysis}

In this section, the inverse-forward displacement analysis of the SPM is approached formulating closure equations based on the components of two unit vectors lying in the plane of the moving platform.

The forward displacement analysis consists of finding the orientation of the moving platform as observed from the fixed platform given the generalized coordinates $q_{i}$ of the parallel manipulator. Said otherwise, it is required to compute the unit vectors $\hat{v}_{i}$, as well as the coordinates of points $B_{i}$. To this aim, let us consider that $\hat{\boldsymbol{v}}_{i}$ is the $i$-th unit vector directed from point $C$ to the $i$-th point $B_{i}$. It is evident that due to the symmetry of the sliding guides, we have a simple closure equation as:

$$
\hat{\boldsymbol{v}}_{1}+\hat{\boldsymbol{v}}_{2}+\hat{\boldsymbol{v}}_{3}=\mathbf{0}
$$

Thereafter, the position vectors of the centers of the spherical joints may be expressed on the unit vectors $\hat{\boldsymbol{v}}_{i}$ as follows:

$$
\boldsymbol{b}_{i}=\boldsymbol{c}+b_{i} \hat{\boldsymbol{v}}_{i}
$$

On the other hand, owing to the assemble of the outer limbs to the fixed platform through concentric revolute joints, it follows that for each outer kinematic chain, its motion is constrained in such a way that necessarily:

$$
\left(\boldsymbol{b}_{i}-\boldsymbol{a}_{i}\right) \cdot \hat{\boldsymbol{u}}_{i}=0
$$

Hence, with the substitution of Equation (2) into Equation (3), it follows that:

$$
b_{i}=a / \hat{\boldsymbol{u}}_{i} \cdot \hat{\boldsymbol{v}}_{i}
$$

Furthermore, based on the magnitude of vector $\boldsymbol{b}_{i}$ and the geometry of the equilateral triangle $O A_{i} B_{i}$, one obtains:

$$
\left(\boldsymbol{c}+b_{i} \hat{\boldsymbol{v}}_{i}\right) \cdot\left(\boldsymbol{c}+b_{i} \hat{\boldsymbol{v}}_{i}\right)=a^{2}+q_{i}^{2}
$$

where $q_{i}$ is the signed distance between $A_{i}$ and $B_{i}$. After a few computations, we have:

$$
\left[\left(\hat{\boldsymbol{u}}_{i} \cdot \hat{\boldsymbol{v}}_{i}\right) \boldsymbol{c}+a \hat{\boldsymbol{v}}_{i}\right] \cdot\left[\left(\hat{\boldsymbol{u}}_{i} \cdot \hat{\boldsymbol{v}}_{i}\right) \boldsymbol{c}+a \hat{\boldsymbol{v}}_{i}\right]-\left(a^{2}+q_{i}^{2}\right)\left(\hat{\boldsymbol{u}}_{i} \cdot \hat{\boldsymbol{v}}_{i}\right)^{2}=0
$$

Expressions (6) lead us to a system of three quadratic equations in six unknowns, e.g., the components of the unit vectors $\hat{\boldsymbol{v}}_{1}$ and $\hat{\boldsymbol{v}}_{2}$. The number of required equations is completed taking into account that:

$$
\hat{\boldsymbol{v}}_{i} \cdot \hat{\boldsymbol{v}}_{i}=1
$$

In the contribution, this system of equations, named the characteristic equations of the SPM, is solved by means of the homotopy continuation method [36]. Afterwards, the coordinates of $B_{i}$ are calculated according to Equation (2). Furthermore, the coordinates of points $D_{i}$, a necessary step for approaching the instantaneous kinematics of the SPM, may be computed based on the following closure equation:

$$
\left(\boldsymbol{d}_{i}-\boldsymbol{b}_{i}\right) \cdot \hat{\boldsymbol{v}}_{i}=0 \rightarrow d_{i}=\boldsymbol{b}_{i} \cdot \hat{\boldsymbol{v}}_{i} / \hat{\boldsymbol{u}}_{i} \cdot \hat{\boldsymbol{v}}_{i}
$$

where $\boldsymbol{d}_{i}=d_{i} \hat{\boldsymbol{u}}_{i}$.

On the other hand, the rotation matrix $\mathrm{R}$ may be computed on the unit vectors $\hat{\boldsymbol{v}}_{1}$ and $\hat{\boldsymbol{v}}_{2}$ as follows:

$$
\mathrm{R}=\left[\begin{array}{ll}
\hat{\boldsymbol{v}}_{1} & \left.\hat{\boldsymbol{v}}_{1} \times \hat{\boldsymbol{v}}_{2} /\left|\hat{\boldsymbol{v}}_{1} \times \hat{\boldsymbol{v}}_{2}\right| \quad \hat{\boldsymbol{v}}_{1} \times\left(\hat{\boldsymbol{v}}_{1} \times \hat{\boldsymbol{v}}_{2}\right) /\left|\hat{\boldsymbol{v}}_{1} \times\left(\hat{\boldsymbol{v}}_{1} \times \hat{\boldsymbol{v}}_{2}\right)\right|\right]
\end{array}\right]
$$


Furthermore, matrix R may be expressed considering conventional roll $(\gamma)$, pitch $(\beta)$ and yaw $(\alpha)$ angles [37] as follows:

$$
\mathbf{R}=\mathbf{R}_{\gamma \beta \alpha}=\left[\begin{array}{ccc}
c(\alpha) c(\beta) & c(\alpha) s(\beta) s(\gamma)-s(\alpha) c(\gamma) & c(\alpha) s(\beta) c(\gamma)+s(\alpha) s(\gamma) \\
s(\alpha) c(\beta) & s(\alpha) s(\beta) s(\gamma)+c(\alpha) c(\gamma) & s(\alpha) s(\beta) c(\gamma)-c(\alpha) s(\gamma) \\
-s(\beta) & c(\beta) s(\gamma) & c(\beta) c(\gamma)
\end{array}\right]
$$

where $s(*)$ and $c(*)$ are the abbreviations of $\sin (*)$ and $\cos (*)$, respectively. Meanwhile, the rotation matrix $\mathrm{R}$ is obtained following the order of rotations of roll, pitch and then yaw. Thereafter, the angles $\alpha, \beta$ and $\gamma$ may be obtained from Equations (9) and (10). Alternatively, the orientation of the moving platform can be also specified considering a unit vector $\hat{\boldsymbol{n}}$ normal to the plane of the moving platform, namely the equivalent angle of finite rotation. Indeed:

$$
\hat{\boldsymbol{n}}=\hat{\boldsymbol{v}}_{1} \times \hat{\boldsymbol{v}}_{2} /\left|\hat{\boldsymbol{v}}_{1} \times \hat{\boldsymbol{v}}_{2}\right|
$$

The vector $\hat{\boldsymbol{n}}$ gives us a clear idea about the orientation of the moving platform.

Finally, the inverse displacement analysis of the robot manipulator is straightforward. In fact, given the orientation of the moving platform, e.g., given the vectors $\hat{\boldsymbol{v}}_{i}$, first the position vectors $\boldsymbol{b}_{i}$ are obtained directly from Equation (2). After, the generalized coordinates $q_{i}$ are computed as follows:

$$
q_{i}^{2}=\left(\boldsymbol{c}+b_{i} \hat{\boldsymbol{v}}_{i}\right) \cdot\left(\boldsymbol{c}+b_{i} \hat{\boldsymbol{v}}_{i}\right)-a^{2}
$$

Note that unlike the forward displacement analysis, a closed-form solution is available for solving the inverse displacement analysis.

It is noteworthy that the orientation of a rigid body in the Euclidean space is represented univocally by a rotation matrix, which is an element of the special orthogonal group denoted by $S O(3)$.

This section introduces a method that does not require the computation of the rotation matrix for solving the forward displacement analysis. The computation of the rotation matrix was included in the contribution only for the sake of completeness.

\section{Input-Output Equation of Velocity}

The velocity analysis of parallel manipulators consists of formulating equations involving the individual kinematic characteristics of their components. The solution of the velocity analysis allows us to measure the angular and linear velocities of any of its elements. A relevant application of velocity analysis includes computing the angular and linear velocities of the moving platform. Formulating the velocity analysis of kinematic chains by means of using screw theory and linear algebra dates back to the 1970s and has been well appreciated by the kinematician community. In this section, the velocity analysis of the SPM is approached by means of the theory of screws [38,39].

Velocity modeling demands formulating a specific linear map between two vector spaces at a given configuration, i.e., velocity modeling involves the linear map between the velocity state, or twist about a screw, and the actuator rates. The velocity state of the moving platform as observed from the fixed platform, notated as a six-dimensional vector $V_{C}$, is defined as follows:

$$
V_{C} \equiv\left[\begin{array}{c}
\omega \\
v_{C}
\end{array}\right]
$$

where $\omega$ is the angular velocity vector of the moving platform as measured from the fixed platform, while $\boldsymbol{v}_{C}$ is the linear velocity vector of point $C$ expressed in the $O_{-} X Y Z$ reference frame. Furthermore, 
the velocity state $V_{C}$ may be written through each limb as a linear combination of the screws representing the kinematic pairs of the $i$-th leg as follows:

$$
{ }_{0} \omega_{1}^{i 0} \$_{i}^{1}+{ }_{1} \omega_{2}^{i 1} \$_{i}^{2}+{ }_{2} \omega_{3}^{i 2} \$_{i}^{3}+{ }_{3} \omega_{4}^{i 3} \$_{i}^{4}+{ }_{4} \omega_{5}^{i 4} \$_{i}^{5}+{ }_{5} \omega_{6}^{i 5} \$_{i}^{6}=V_{C}
$$

where the assignation of screws is as follows: ${ }^{0} \$_{i}^{1}$ denotes the screw associated with the lower revolute joint connecting the limb to the fixed platform, ${ }^{1} \$_{i}^{2}$ denotes the screw representing the actuated prismatic joint along the limb, the screws ${ }^{2} \$_{i}^{3},{ }^{3} \$_{i}^{4}$ and ${ }^{4} \$_{i}^{5}$ are screws with concurrent primal parts reproducing the effect of the spherical joint and ${ }^{5} \$_{i}^{6}$ represents the screw associated with the upper passive prismatic joint. On the other hand, with the purpose of simplifying the analysis, let us consider that $S_{i}$ is a line in Plücker coordinates directed from $B_{i}$ to $D_{i}$. Note that this line is reciprocal to all the screws in the same limb except the screw representing the actuated prismatic joint. Let $\$_{1}=\left(\hat{\mathbf{s}}_{1}, \boldsymbol{s}_{\mathrm{O}}\right)$ and $\$_{2}=\left(\hat{\mathbf{s}}_{2}, \boldsymbol{s}_{\mathrm{O} 2}\right)$ be two elements of the Lie algebra se(3) of the Euclidean group $S E(3)$. The Klein form, notated as $\{* ; *\}$, is a bilinear symmetric form defined as follows:

$$
\left\{\$_{1} ; \$_{2}\right\}=\hat{\boldsymbol{s}}_{1} \cdot \boldsymbol{s}_{\mathrm{O} 2}+\hat{\mathbf{s}}_{2} \cdot \boldsymbol{s}_{\mathrm{O} 1}
$$

Furthermore, it is said that the screws $\$_{1}$ and $\$_{2}$ are reciprocal if $\left\{\$_{1} ; \$_{2}\right\}=0$. Thus, the application of the Klein form between the line $S_{i}$ and both sides of Equation (14) with the cancellation of terms leads to:

$$
*\left\{S_{i} ; V_{C}\right\}=\dot{q}_{i}\left\{S_{i} ;{ }^{1} \$_{i}^{2}\right\}
$$

where $\dot{q}_{i}={ }_{1} \omega_{2}^{i}$ is the $i$-th generalized speed. Expression (15) provides enough information to obtain the input-output equation of velocity. However, the handling of non-square Jacobian matrices is imminent. The expansion of the Jacobians of limited-dof parallel manipulators to full rank Jacobian matrices brings important advantages [40]. Dealing with the contribution, in order to avoid the tedious handling of non-square matrices, consider that a standard basis for parallel manipulators performing the spherical motion is given by $\left\{\$_{1}, \$_{2}, \$_{3}\right\}$ where $\$_{1}=[\hat{i}, 0], \$_{2}=[\hat{j}, 0]$ and $\$_{3}=[\hat{k}, 0]$. Therefore, by resorting to the concept of the reciprocal screw through the Klein form, three equations may be written as follows:

$$
\left\{\$_{i} ; V_{C}\right\}=0
$$

Casting into a matrix-vector form Expressions (15) and (16), the input-output equation of the velocity of the SPM results in:

$$
\mathrm{J}_{\mathrm{v}}^{\mathrm{T}} \Delta V_{\mathrm{C}}=\mathrm{J}_{\mathrm{q}} \mathrm{Q}_{\mathrm{v}}
$$

where

$\mathrm{J}_{\mathrm{v}}=\left[\begin{array}{llllll}S_{1} & S_{2} & S_{3} & \$_{1} & \$_{2} & \$_{3}\end{array}\right]$ is the forward Jacobian matrix,

$\mathrm{J}_{\mathrm{q}}=\operatorname{diag}\left[\left\{S_{1} ;{ }^{1} \$_{1}^{2}\right\} \quad\left\{S_{2} ;{ }^{1} \$_{2}^{2}\right\} \quad\left\{S_{3} ;{ }^{1} \$_{3}^{2}\right\} \quad 1 \quad 1 \quad 1 \quad 1\right]$ is the inverse Jacobian matrix,

$\mathrm{Q}_{\mathrm{v}}=\left[\begin{array}{llllll}\dot{q}_{1} & \dot{q}_{2} & \dot{q}_{3} & \dot{q}_{4} & \dot{q}_{5} & \dot{q}_{6}\end{array}\right]^{\mathrm{T}}$ where $\dot{q}_{4}=\dot{q}_{5}=\dot{q}_{6}=0$ is the first order driver matrix and

$\Delta=\left[\begin{array}{ll}0 & \mathrm{I} \\ \mathrm{I} & 0\end{array}\right]$ is an operator of polarity generated with the zero matrix 0 and the identity matrix I.

\section{Input-Output Equation of Acceleration}

Acceleration analysis consists of formulating equations involving the angular and linear accelerations of each element of a parallel manipulator. The solution of acceleration analysis allows us to determine the angular and linear accelerations of any body of the system under study. Pioneering contributions modeling the acceleration of rigid bodies based on six-dimensional vectors date back to 1947 when Brand introduced in kinematics the concept of the acceleration motor or, (for brevity, accelerator). However, while the velocity analysis in screw form of robot manipulators had been 
welcome almost five decades ago in the kinematician community, still these days, there is certain incredulity about the validity of the equations in screw form of the acceleration analysis. In this section, the acceleration analysis by means of screw theory of the spherical parallel manipulator is briefly presented [39].

Let $\boldsymbol{\alpha}$ be the angular acceleration vector of the moving platform as measured from the fixed platform. Furthermore, let $\boldsymbol{a}_{c}$ be the acceleration of point $C$ expressed in the $O_{-} X Y Z$ fixed reference frame. The reduced acceleration state, or accelerator, for brevity, of the moving platform as observed from the fixed platform, a six-dimensional vector notated as $A_{C}$, is defined as follows:

$$
A_{C}=\left[\begin{array}{c}
\boldsymbol{\alpha} \\
a_{C}-\boldsymbol{\omega} \times \boldsymbol{v}_{C}
\end{array}\right]
$$

Furthermore, the accelerator may be expressed in screw form through the external limbs as follows:

$$
{ }_{0} \alpha_{1}^{i 0} \$_{i}^{1}+{ }_{1} \alpha_{2}^{i 1} \$_{i}^{2}+{ }_{2} \alpha_{3}^{i 2} \$_{i}^{3}+{ }_{3} \alpha_{4}^{i 3} \$_{i}^{4}+{ }_{4} \alpha_{5}^{i 4} \$_{i}^{5}+{ }_{5} \alpha_{6}^{i 5} \$_{i}^{6}+L_{i}=A_{C}
$$

where $L_{i}$ is the Lie screw of acceleration, which is given by:

$$
\begin{aligned}
& L_{i}=\left[{ }_{0} \omega_{1}^{i 0} \$_{i}^{1} \quad{ }_{1} \omega_{2}^{i 1} \$_{i}^{2}+{ }_{2} \omega_{3}^{i 2} \$_{i}^{3}+{ }_{3} \omega_{4}^{i 3} \$_{i}^{4}+{ }_{4} \omega_{5}^{i 4} \$_{i}^{5}+{ }_{5} \omega_{6}^{i 5} \$_{i}^{6}\right]+ \\
& {\left[{ }_{1} \omega_{2}^{i 1} \$_{i}^{2} \quad{ }_{2} \omega_{3}^{i 2} \$_{i}^{3}+{ }_{3} \omega_{4}^{i 3} \$_{i}^{4}+{ }_{4} \omega_{5}^{i 4} \$_{i}^{5}+{ }_{5} \omega_{6}^{i 5} \$_{i}^{6}\right]+} \\
& {\left[{ }_{2} \omega_{3}^{i 2} \$_{i}^{3} \quad{ }_{3} \omega_{4}^{i 3} \$_{i}^{4}+{ }_{4} \omega_{5}^{i 4} \$_{i}^{5}+{ }_{5} \omega_{6}^{i 5} \$_{i}^{6}\right]+\left[{ }_{3} \omega_{4}^{i 3} \$_{i}^{4} \quad{ }_{4} \omega_{5}^{i 4} \$_{i}^{5}+{ }_{5} \omega_{6}^{i} \$_{i}^{6}\right]+} \\
& {\left[{ }_{4} \omega_{5}^{i 4} \$_{i}^{5} \quad{ }_{5} \omega_{6}^{i 5} \$_{i}^{6}\right]}
\end{aligned}
$$

Following the framework of the velocity analysis, the input-output equation of acceleration results in:

$$
\mathrm{J}_{\mathrm{v}}^{\mathrm{T}} \Delta A_{\mathrm{C}}=\mathrm{J}_{\mathrm{q}} \mathrm{Q}_{\mathrm{a}}+\mathrm{C}
$$

Therein:

$\mathrm{Q}_{\mathrm{a}}=\left[\begin{array}{llllll}\ddot{q}_{1} & \ddot{q}_{2} & \ddot{q}_{3} & \ddot{q}_{4} & \ddot{q}_{5} & \ddot{q}_{6}\end{array}\right]^{\mathrm{T}}$ where $\ddot{q}_{4}=\ddot{q}_{5}=\ddot{q}_{6}=0$ is the second-order driver matrix and $C=\left[\begin{array}{llllll}\left\{S_{1} ; L_{1}\right\} & \left\{S_{2} ; L_{2}\right\} & \left\{S_{3} ; L_{3}\right\} & 0 & 0 & 0\end{array}\right]^{\mathrm{T}}$ is the complementary matrix of acceleration.

It is interesting to note that owing to the fact that the dual part of the accelerator $A_{C}$ vanishes, then matrix $C$ can be removed from Equation (21). The explanation is easy to follow. It is evident that the terms of the Coriolis acceleration are contained in the dual part of $A_{C}$ and in the Lie screw of acceleration (see Equation (19)); thus, it follows that if $\boldsymbol{a}_{C}-\boldsymbol{\omega} \times \boldsymbol{v}_{C}=\mathbf{0}$, then necessarily, $\boldsymbol{L}_{i}=\mathbf{0}$. Said otherwise, in an SPM, the reduced acceleration state of the moving platform as measured from the fixed platform, taking the point of null linear motion of the moving platform as the reference pole, is generated only based on a linear combination of the joint acceleration rates of the $i$-th limb.

\section{Numerical Applications}

In order to show the application of the method, in this section, the numerical kinematic analysis of the spherical parallel manipulator under study is presented. To this aim, consider that the parameters of the mechanism are chosen as $h=1 \mathrm{~m}, a=1 \mathrm{~m}, \hat{\boldsymbol{u}}_{1}=\hat{\boldsymbol{i}}, \hat{\boldsymbol{u}}_{2}=-0.5 \hat{\boldsymbol{i}}-0.866 \hat{\boldsymbol{k}}$ and $\hat{\boldsymbol{u}}_{3}=-0.5 \hat{\boldsymbol{i}}+0.866 \hat{\boldsymbol{k}}$. Thus, the coordinates of the nominal positions of the lower revolute joints are given by $A_{1}=(1,0,0) \mathrm{m}$, $A_{2}=(-0.5,0,-0.866) \mathrm{m}$ and $A_{3}=(-0.5,0,0.866) \mathrm{m}$.

The first part of the numerical example deals with the inverse displacement analysis of the SPM. To this aim, let us consider that the orientation of the moving platform is defined by the angles roll $\left(\gamma=3^{\circ}\right)$, pitch $\left(\beta=2^{\circ}\right)$ and yaw $\left(\alpha=4^{\circ}\right)$. Then, according to Equation (12), the generalized coordinates meeting this pose result in $q_{1}=0.967524 \mathrm{~m}, q_{2}=1.06524 \mathrm{~m}$ and $q_{3}=0.974468 \mathrm{~m}$. 
The next part of the case study deals with the forward displacement analysis of the SPM. The application consists of finding all the feasible poses of the moving platform given the generalized coordinates $q_{i}$ obtained for the inverse displacement analysis of the SPM. The method introduced in Section 3 yields 60 solutions where only four are real. In that regard, the available values for vectors $\hat{\boldsymbol{v}}_{1}$ and $\hat{\boldsymbol{v}}_{2}$ are listed in Table 1.

Table 1. Numerical forward displacement analysis: resulting unit vectors $\hat{\boldsymbol{v}}_{1}$ and $\hat{\boldsymbol{v}}_{2}$.

\begin{tabular}{ccc}
\hline Sol. & $\hat{v}_{1}$ & $\hat{v}_{2}$ \\
\hline 13 & $(-0.9969563613,0.0348994966,0.0697139786)$ & $(0.5572283259,-0.0627464056,0.8279851940)$ \\
16 & $(-0.9970863751,0.0347732475,-0.0678939009)$ & $(0.4382165437,-0.0627290151,0.8966779419)$ \\
45 & $(0.9970863751,-0.0347732475,0.0678939009$ & $(-0.4382165437,0.0627290151,-0.8966779419)$ \\
48 & $(0.9969563613,-0.0348994966,-0.0697139786)$ & $(-0.5572283259,0.0627464056,-0.8279851940)$ \\
\hline
\end{tabular}

In what follows, the method is proven solving numerically the velocity and acceleration analyses of the robot manipulator. To this end, consider Solution 45 of Table 1 as the reference configuration of the parallel manipulator. For clarity, in Table 2, the data of the home position of the SPM are provided.

Table 2. Reference configuration of the spherical parallel manipulator.

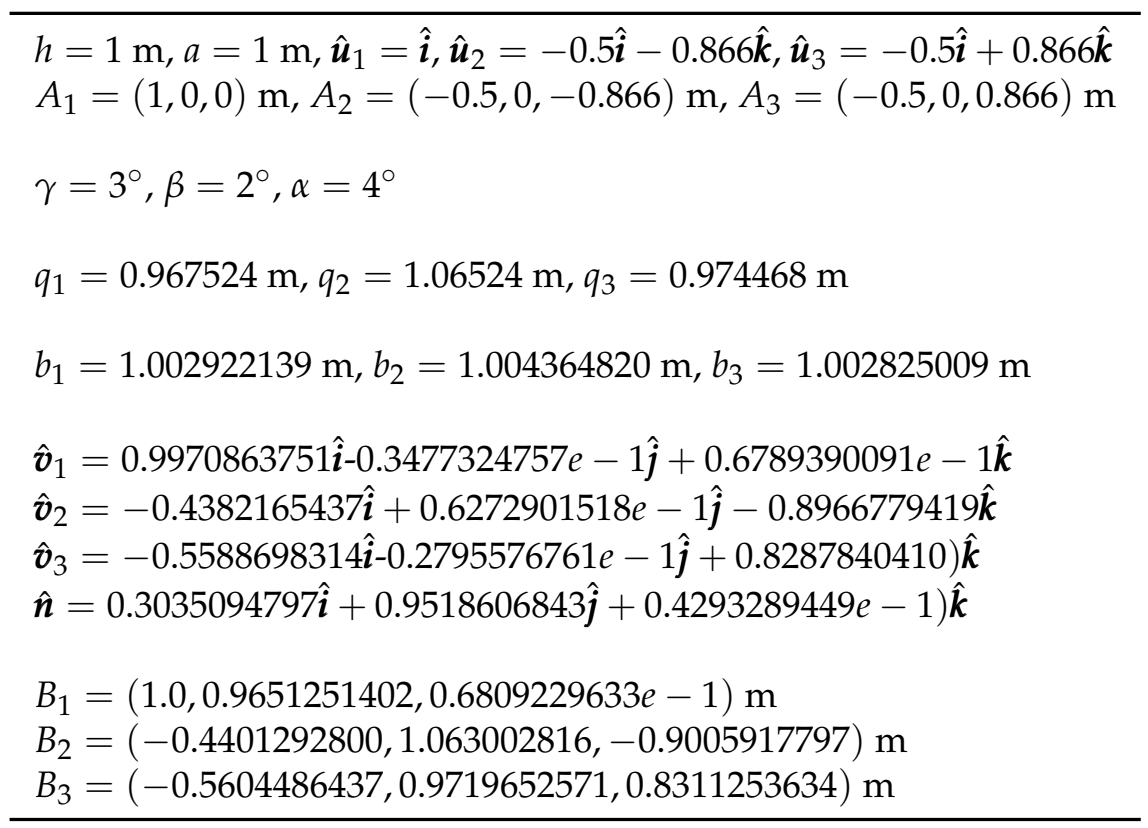

With these assumptions, based on the reference configuration of the robot, the moving platform must reach an orientation defined by the angles $\gamma=20^{\circ}, \beta=60^{\circ}, \alpha=30^{\circ}$. Furthermore, at the beginning of the analysis, the parallel manipulator is motionless, and it is required to move it in a smooth manner in such a way that after 10 seconds, the moving platform returns to rest, reaching the desired orientation. A quintic polynomial expression is appropriated to achieve this task. For clarity, plots of the functions of the generalized coordinates and their time derivatives meeting such conditions are provided in Figure 2. 


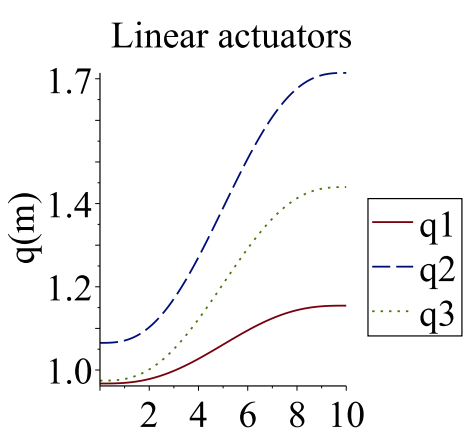

$\mathrm{t}(\mathrm{s})$

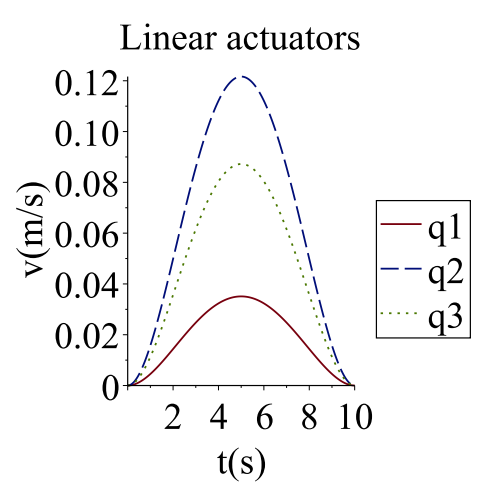

Figure 2. Time history of the generalized coordinates. Application 1.

The application of the proposed method yields the temporal behavior of the angular velocity and acceleration of the moving platform shown in Figure 3.
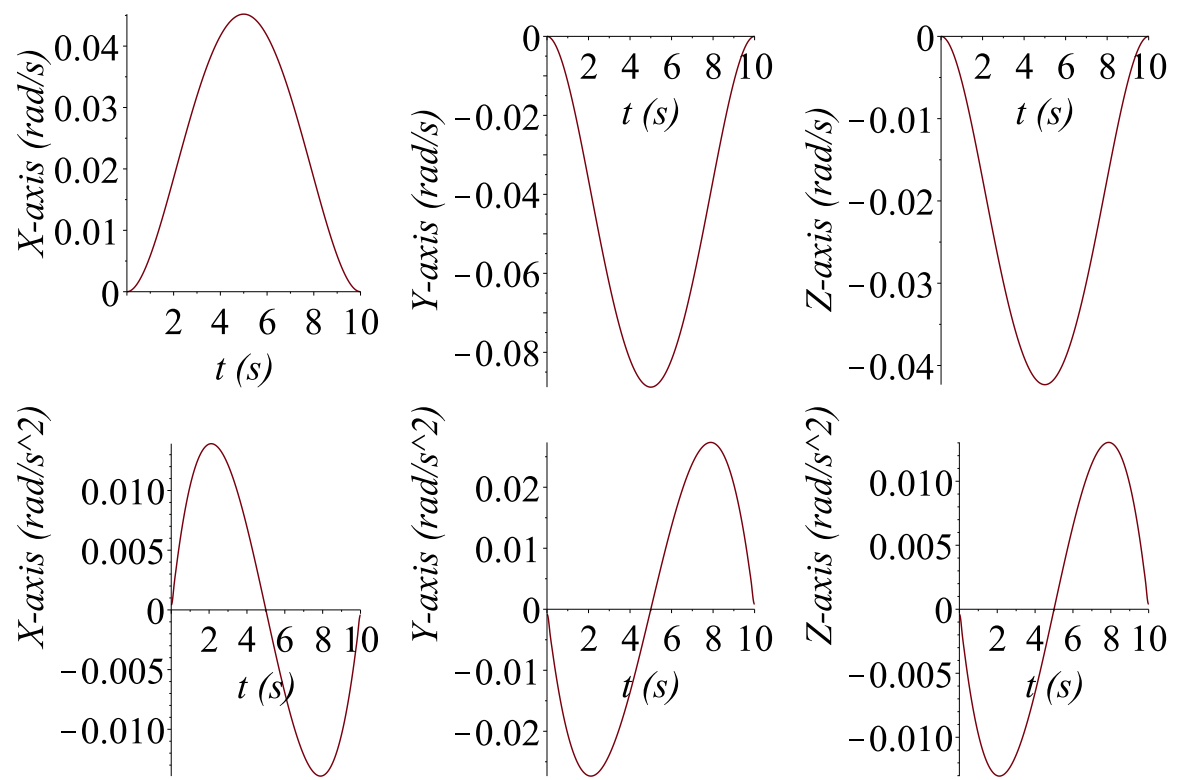

Figure 3. Instantaneous kinematics of the moving platform. Application 1.

It is interesting to mention that the components of the angular acceleration of the moving platform may be obtained using another approach, namely Method 2. This alternative consists of two steps. First, the components of the angular velocity vector generated by applying screw theory are transformed into analytic functions using spline functions. Second, the components of the angular acceleration vector are obtained as simple time derivatives of the functions generated in the first step. Figure 4 shows a comparison of both strategies.

Note that according to Figure 4, the numerical results of the computation of the angular acceleration of the moving platform by using screw theory are in excellent agreement with those generated with Method 2.

A second application concerned with the velocity and acceleration analysis of the SPM is included in the contribution. Based on the reference configuration of the parallel manipulator given in Table 2, the generalized coordinates are commended to follow periodical functions given by $q_{1}=0.25 s(t) c(t)$, $q_{2}=-0.75 s(t) c(t)$ and $q_{3}=0.5 s(t) c(t)$ where the time $t$ is given in the interval $0 \leq t \leq 2 \pi \mathrm{s}$. The resulting temporal behavior of the kinematics of the moving platform of this case is provided in Figure 5. 

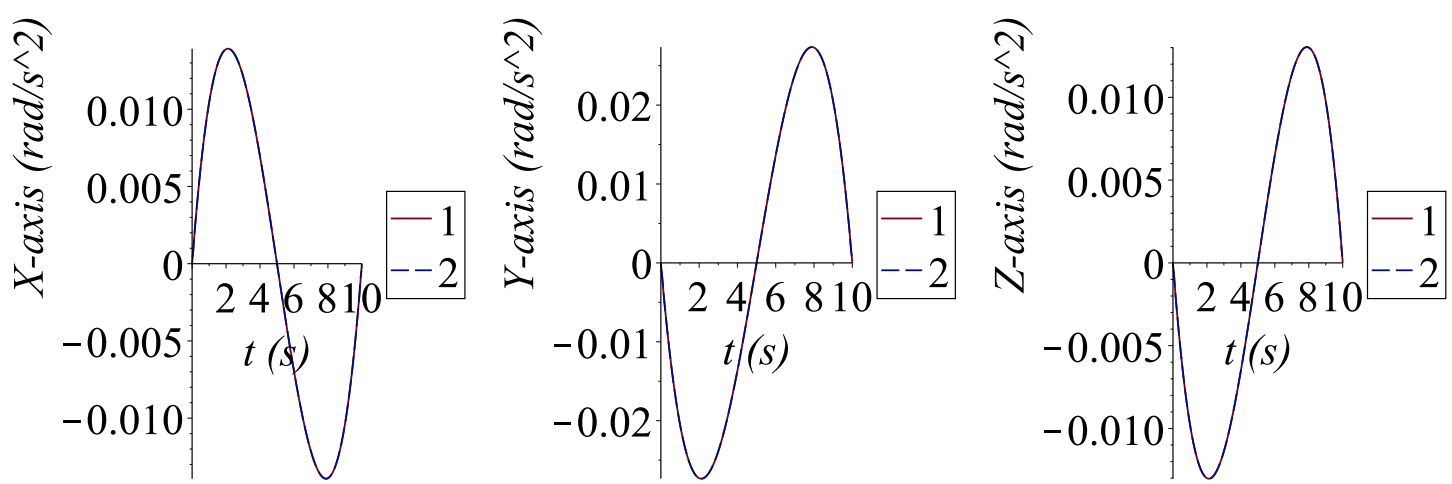

Figure 4. Two methods for computing the angular acceleration vector of the moving platform: screw theory (1), Method 2 (2). Application 1.
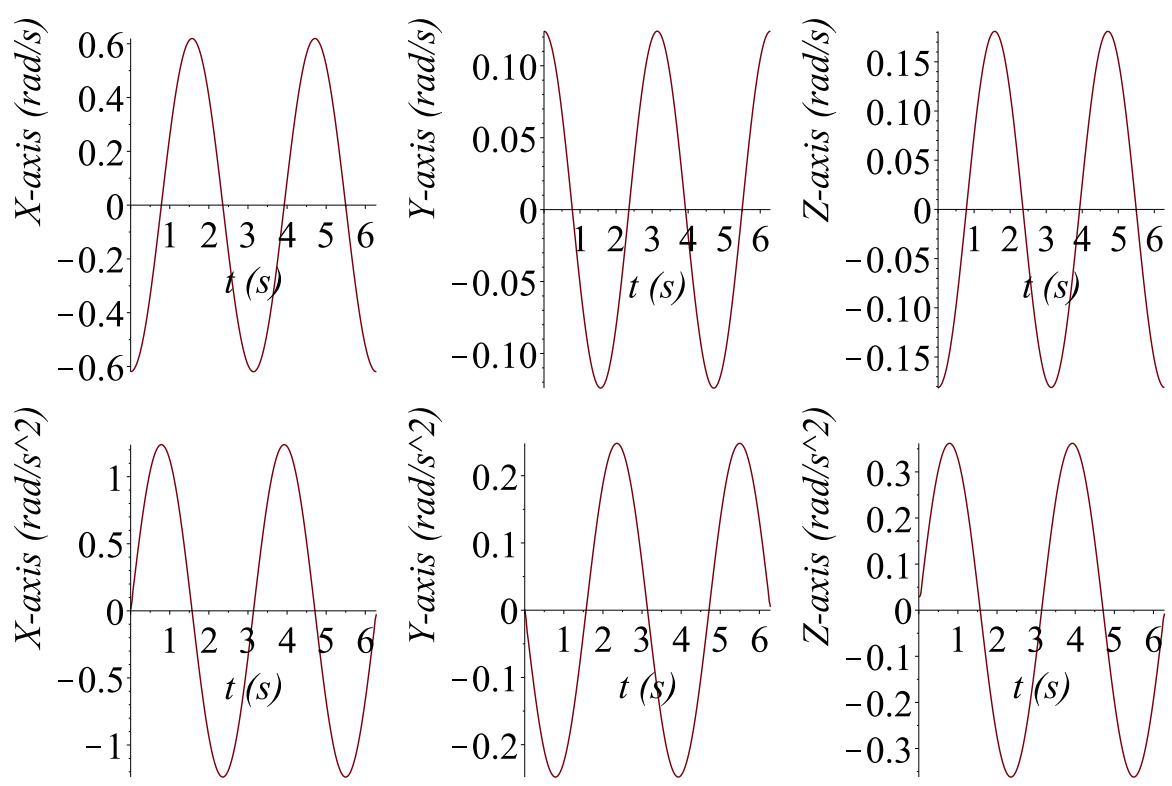

Figure 5. Instantaneous kinematics of the moving platform. Application 2.

Meanwhile, the comparison of the computation of the angular acceleration vector using screw theory and Method 2 is shown in Figure 6.
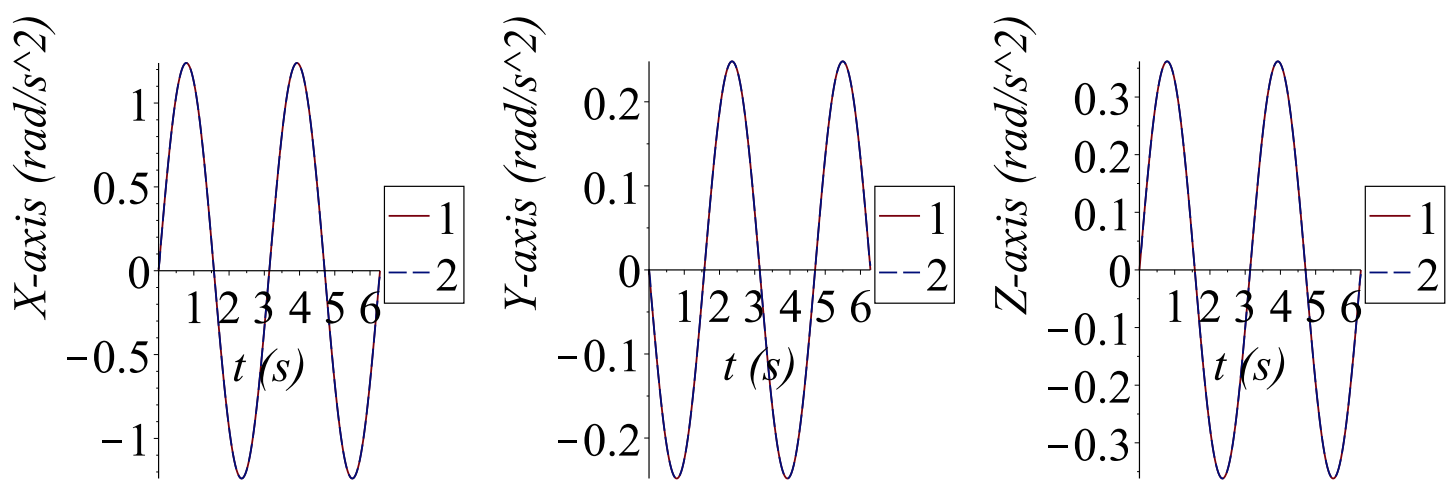

Figure 6. Two methods for computing the angular acceleration vector of the moving platform: screw theory (1), Method 2 (2). Application 2. 


\section{Conclusions}

In this work, the kinematics of the spherical parallel manipulator introduced by Enferadi and Shahi [35] is approached by means of the theory of screws. This parallel manipulator is composed of three active peripheral limbs and a passive center shaft.

The displacement analysis of the SPM at hand was carried out formulating closure equations based on simple linear combinations of two unit vectors describing the orientation of the moving platform as observed from the fixed platform. The method is easy to follow and does not require the computation of the rotation matrix for solving the forward displacement analysis, a recursive strategy employed in most contributions approaching the displacement analysis of parallel manipulators. The computation of the rotation matrix based on the roll, pitch and yaw angles was included only for the sake of completeness. Afterwards, the input-output equations of velocity and acceleration of the SPM were achieved by resorting to reciprocal-screw theory. This method does not require the computation of the passive joint velocity and acceleration rates of the SPM, without a doubt an interesting advantage of the proposed method. Finally, numerical examples were included with the purpose to illustrate the application of the method.

Author Contributions: The displacement analysis of the SPM was carried out by R.R.-C. and L.P.-G. J.G.-A. realized the velocity and acceleration analyses of the SPM, as well as the solution of the numerical examples. C.R.A.-N. performed the translation of the mathematical framework developed for the kinematics of the SPM into computer codes. J.G.-A. is the first author of the contribution.

Funding: This research was funded by Conacyt grant number 7903.

Acknowledgments: The first author acknowledge with thanks the support of the National Council of Science and Technology of Mexico (CONACYT) through the National Network of Researchers fellowship; Grant Number 7903.

Conflicts of Interest: The authors declare no conflict of interest.

\section{References}

1. Peres, A. Finite rotations and angular velocity. Am. J. Phys. 1980, 48, 70-71. [CrossRef]

2. Ting, K.-L.; Soni, A.H. Instantaneous kinematics of a plane in spherical motion. ASME J. Mech. Trans. Autom. Des. 1983, 105, 560-567. [CrossRef]

3. Nikravesh, P.E.; Wehage, R.A.; Kwon, O.K. Euler parameters in computational kinematics and dynamics. Part 1. ASME J. Mech. Trans. Autom. Des. 1985, 107, 358-365. [CrossRef]

4. $\quad$ Angeles, J. Rational Kinematics; Springer: New York, NY, USA, 1988.

5. Chiang, C.H. Kinematics of Spherical Mechanisms; Cambridge University Press: Cambridge, UK, 1988.

6. Rico-Martinez, J.M.; Gallardo-Alvarado, J. A simple method for the determination of angular velocity and acceleration of a spherical motion through quaternions. Meccanica 2000, 35, 111-118. [CrossRef]

7. Chase, R.; Pandya, A. A Review of active mechanical driving principles of spherical robots. Robotics 2012, 1, 3-23. [CrossRef]

8. McCarthy, J.M.; Bodduluri, R.M. Avoiding singular configurations in finite position synthesis of spherical 4R linkages. Mech. Mach. Theory 2000, 35, 451-462. [CrossRef]

9. McCarthy, J.M.; Soh, G.S. Geometric Design of Linkages, 2nd ed.; Springer: Berlin, Germany, 2011.

10. Cervantes-Sánchez, J.J.; Medellín-Castillo, H.I.; Rico-Martínez, J.M.; González-Galván, E.J. Some improvements on the exact kinematic synthesis of spherical $4 \mathrm{R}$ function generators. Mech. Mach. Theory 2009, 44, 103-121. [CrossRef]

11. Yang, T.; Jian, Y.H.; Lai, R.Y. A Simple Synthesis Method of Spherical 4R Linkages Reaching Four Positions. Adv. Mater. Res. 2011, 199-200, 1236-1239. [CrossRef]

12. Mendoza-Trejo, O.; Cruz-Villara, C.A.; Peón-Escalante, R.; Zambrano-Arjona, M.A.; Peñuñuri, F. Synthesis method for the spherical 4R mechanism with minimum center of mass acceleration. Mech. Mach. Theory 2015, 93, 53-64. [CrossRef]

13. Asada, H.; Granito, J. Kinematic and static characterization of wrist joints and their optimal design. In Proceedings of the IEEE International Conference on Robotics and Automation, St. Louis, MO, USA, 25-28 March 1985; pp. 244-250. 
14. Lo, H.S.; Xie, S. Optimization and analysis of a redundant $4 \mathrm{R}$ spherical wrist mechanism for a shoulder exoskeleton. Robotica 2014, 32, 1191-1211. [CrossRef]

15. Gosselin, C.M.; Hamel, J.F. The Agile Eye: A high-performance three-degree-of-freedom camera-orienting device. In Proceedings of the IEEE International Conference on Robotics and Automation, San Diego, CA, USA, 8-13 May 1994; pp. 781-786.

16. Gosselin, C.M.; St-Pierre, É. Development and experimentation of a fast 3-DOF camera-orienting device. Int. J. Robot. Res. 1997, 16, 619-630 [CrossRef]

17. Li, T.; Payandeh, S. Design of spherical parallel mechanisms for application to laparoscopic surgery. Robotica 2002, 20, 133-138. [CrossRef]

18. Hong, M.B.; Jo, Y.-H. Design of a novel 4-DOF wrist-type surgical instrument with enhanced rigidity and dexterity. IEEE ASME Trans. Mechatron. 2014, 19, 500-511. [CrossRef]

19. Erdman, A.G. Lessons learned from kinematics research applied to medical device design. ASME J. Biomech. Eng. 2018, 140, 021006-1-15. [CrossRef] [PubMed]

20. Gosselin, C.M.; Angeles, J. The optimum kinematic design of a spherical three-degree-of-freedom parallel manipulator. ASME J. Mech. Transm. Autom. Des. 1989, 111, 202-207. [CrossRef]

21. Gosselin, C.M.; Angeles, J. A global performance index for the kinematic optimization of robotic manipulators. ASME J. Mech. Des. 1991, 113, 220-226. [CrossRef]

22. Alici, G.; Shirinzadeh, B. Topology optimisation and singularity analysis of a 3-SPS parallel manipulator with a passive constraining spherical joint. Mech. Mach. Theory 2004, 39, 215-235. [CrossRef]

23. Bonev, I.A.; Gosselin, C.M. Singularity loci of spherical parallel mechanisms. In Proceedings of the IEEE International Conference on Robotics and Automation, Barcelona, Spain, 18-22 April 2005; pp. 2957-2962.

24. Bai, S.; Hansen, M.R.; Andersen, T.O. Modelling of a special class of spherical parallel manipulators with Euler parameters. Robotica 2009, 27, 161-170. [CrossRef]

25. Bai, S. Optimum design of spherical parallel manipulator for a prescribed workspace. Mech. Mach. Theory 2010, 45, 200-211. [CrossRef]

26. Kong, X.; Gosselin, C. A formula that produces a unique solution to the forward displacement analysis of a quadratic spherical parallel manipulator: The agile eye. ASME J. Mech. Robot. 2010, 2, 044501. [CrossRef]

27. $\mathrm{Wu}, \mathrm{G}$. Multiobjective optimum design of a 3-RRR spherical parallel manipulator with kinematic and dynamic dexterities. Model. Identif. Control 2012, 33, 111-121. [CrossRef]

28. Saafi, H.; Laribi, M.; Zeghloul, S. Redundantly actuated 3-RRR spherical parallel manipulator used as a haptic device: Improving dexterity and eliminating singularity. Robotica 2015, 33, 1113-1130. [CrossRef]

29. Shintemirov, A.; Niyetkaliyev, A.; Rubagotti, M. Numerical optimal control of a spherical parallel manipulator based on unique kinematic solutions. IEEE ASME Trans. Mechatron. 2016, 21, 98-109. [CrossRef]

30. Wu, G.; Caro, S.; Wang, J. Design and transmission analysis of an asymmetrical spherical parallel manipulator. Mech. Mach. Theory 2015, 94, 119-131. [CrossRef]

31. Saafi, H.; Laribi, M.A.; Zeghloul, S. Optimal torque distribution for a redundant 3-RRR spherical parallel manipulator used as a haptic medical device. Robot. Auton. Syst. 2017, 89, 40-50. [CrossRef]

32. Arrouk, K.A.; Bouzgarrou, B.C.; Gogu, G. Workspace characterization and kinematic analysis of general spherical parallel manipulators revisited via graphical based approaches. Mech. Mach. Theory 2018, 122, 404-431. [CrossRef]

33. Landuré, J.; Gosselin, C. Kinematic analysis of a novel kinematically redundant spherical parallel manipulator. ASME J. Mech. Robot. 2018, 10, 021007. [CrossRef]

34. Li, X.; Liu, J.; Chen, W.; Bai, S. Integrated design, modeling and analysis of a novel spherical motion generator driven by electromagnetic principle. Robot. Auton. Syst. 2018, in press. [CrossRef]

35. Enferadi, J.; Shahi, A. A closed-form solution for the position analysis of a novel fully spherical parallel manipulator. Robotica 2015, 33, 2114-2136. [CrossRef]

36. Gallardo-Alvarado, J.; Abedinnasab, M.H.; Lichtblau, D. Simplified kinematics for a parallel manipulator generator of the Schönflies motion. ASME J. Mech. Robot. 2016, 8, 061020. [CrossRef]

37. Craig, J.J. Introduction to Robotics: Mechanics \& Control; Addison-Wesley Publishing Company: Boston, MA, USA, 1986.

38. Joshi, S.A.; Tsai, L.W. Jacobian analysis of limited-DOF parallel manipulators. ASME J. Mech. Des. 2002, 124, 254-258. [CrossRef] 
39. Gallardo-Alvarado, J. Kinematic Analysis of Parallel Manipulators by Algebraic Screw Theory; Springer International Publishing: Berlin, Germany, 2016.

40. Choi, H.-B.; Ryu, J. Singularity analysis of a four degree-of-freedom parallel manipulator based on an expanded $6 \times 6$ Jacobian matrix. Mech. Mach. Theory 2012, 57, 51-61. [CrossRef]

(C) 2018 by the authors. Licensee MDPI, Basel, Switzerland. This article is an open access article distributed under the terms and conditions of the Creative Commons Attribution (CC BY) license (http://creativecommons.org/licenses/by/4.0/). 